\title{
An Improved Articulated Model of the Human Hand
}

\author{
John McDonald, Jorge Toro, Karen Alkoby, Andre Berthiaume, Pattaraporn Chomwong, \\ Juliet Christopher, Mary Jo Davidson, Jacob Furst, Brian Konie, Glenn Lancaster, \\ Steven Lytinen, Lopa Roychoudhuri, Eric Sedgwick, Noriko Tomuro, Rosalee Wolfe
}

\author{
School of Computer Science, Telecommunications and Information Systems \\ DePaul University \\ Chicago, IL \\ asl@cs.depaul.edu
}

\begin{abstract}
We present an improved anatomically based approach to modeling the human hand for use in the animation of American Sign Language. The joint rotations in the model are based on the bone and muscle configurations of the hand, and a forward kinematic solution is used to position the hand. In particular, we investigate the rotations of the base joint of the thumb. This joint is a saddle joint with non-trivial rotational axes and centers, and must be treated with care in such a model.

We take advantage of several correlations between joint rotations in the hand to reduce the number of degrees of freedom in the model and provide a simple, intuitive and interactive interface for American Sign Language handshape transcription.
\end{abstract}

Keywords: Modeling, Animation, American Sign Language, Anatomically Based Modeling

\section{INTRODUCTION}

The present work has arisen out of a need to produce recognizable animations of American Sign Language (ASL). ASL is a natural language used by members of the North American Deaf community and is the third or fourth most widely used language in the United States [Stern96]. At present deaf people rely on sign language interpreters for access to spoken English, but cost, availability and privacy issues make this an awkward solution at best. A digital sign language interpreter, which translates spoken English into ASL, would better bridge the gulf between deaf and hearing worlds.

Current technology for the translation of written English includes closed captioning on television and TDD. These are good first efforts at making spoken English more accessible to the deaf population, but do not represent a completely satisfactory solution. While ASL shares some vocabulary with English, there is no simple word-for-word translation. Further, research in linguistics shows that ASL's concise and elegant syntax differs radically from English grammar [Klima 79][Valli93]. Because of the differences in the two languages, most native ASL signers read English at the third or fourth grade level [Holt94]. Again, a digital sign language interpreter, which translates written English into ASL, would better aid the deaf community.

In ASL, the shape of the hand is a key determinant of a sign's meaning, and the standard for judging specific handshapes is high. Often a slight change in a feature of a handshape will render it unrecognizable.

ASL also presents a need for efficient, realistic techniques for handshape transcriptions for use in animation. We are building a lexical database for ASL with the goal of working with a group of native ASL signers who will transcribe the signs. ASL signers are not necessarily trained in mathematics or computer graphics, and few are willing to invest the large amount of time necessary to learn a generalized animation package.

The human hand, like most articulated figures, can be modeled as a collection of articulated rigid bodies connected by joints with one or more degrees of rotational freedom. While the body of work on articulated figures is large and varied [Badle99] [Zhao94][Earns98], the number of studies of the hand is relatively small when compared to the literature on human motion. When modeling the human figure for ergonomic studies or for virtual stunt doubles in film, the gross behavior of human 
motion is the key element, and subtle features like the hand can remain simplistic [Badle99].

The main body of work in computer animation of the human hand has revolved around the ability of an animated character to grasp objects effectively and convincingly [Rijpk91]. However the act of grasping does not encompass the full range of expressiveness that a human hand can achieve. This work has limited usefulness for other applications such as depicting ASL because the hand is almost always in an open configuration necessary to encompass the object, see Figure 1. Also, the approximation of the thumb used in many grasping models does not accurately reflect the motions of the human thumb [Gir91].

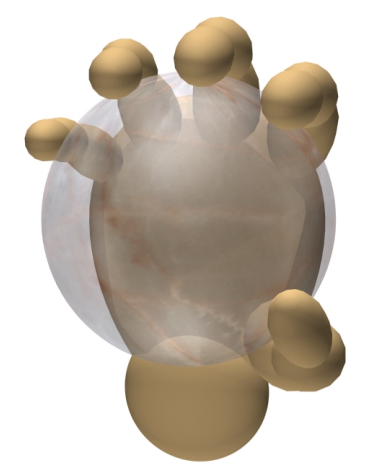

Figure 1: Our model in an open position for grasping.

Related work in robotics also addresses grasping. However, the main purpose of most robot arms is to manipulate machinery, not to reproduce convincingly the possible shapes of the human hand. Few robotic hands mimic the mechanics of a human hand, and many have fewer than five digits. One notable exception is the work of David Jaffe on creating a mechanical finger spelling hand for the Deaf-Blind [Jaffe89].

Other work on modeling the human hand has centered on modeling deformations of both the skin and external objects during a grasping task [Gourr89]. Realistic skin deformations such as those described in [Gourr89] are definitely desirable for realism in many applications but the computational cost of such models is still prohibitive for many realtime animations.

We desired an efficient anatomically based model of the joints in the human hand which simulated realistic joint rotations and could be manipulated with a natural set of interactive controls. The geometry of the model and the user interface should accurately reflect the basic motions of the human fingers and thumb. Since we were primarily interested in the final shape of the hand and not in the precise points of contact with other objects, we could use a forward kinematic solution.
As we shall see, the geometry of the joints in the hand must be specified with care to achieve natural motions in the hand while minimizing unneeded degrees of freedom in the model. Our model contains several key improvements, which enhance the hand's expressiveness while preserving simplicity in the model and allowing for an intuitive interface.

\section{THE ANATOMY OF THE HUMAN HAND}

We begin with a short anatomical description of the hand. For a complete description, see [Caill82], [Lands55], or [Nette87]. The base of the hand consists of a collection of small carpal bones, see Figure 2. Because the motions of these bones are primarily related to the flexion and abduction of the wrist, we will ignore most considerations of the carpals in this work, with one exception. The carpal that articulates with the base of the thumb is called the trapezium, and its shape has an enormous effect on the motion of the thumb.

The main body of the palm is composed of four long bones called the metacarpals which articulate with the carpals in a collection of joints known as the carpometacarpal (CMC) joints.

Each metacarpal bone forms the base of a finger. An additional and far more mobile metacarpal bone forms the base of the thumb, and articulates with the trapezium.

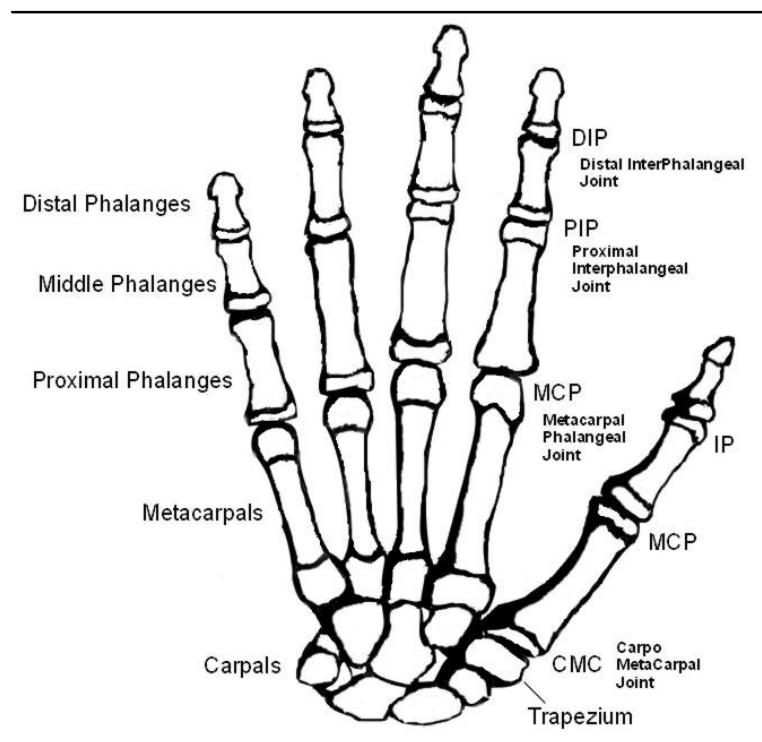

Figure 2: The bones and joints of the human hand

The second and third metacarpal bones, for the index and middle fingers respectively, are fixed, while the metacarpals of the ring and pinky fingers are free to move in a small range. Metacarpals four and five account for some motion in the fingers and for deformations in the palm itself, but we will ignore the contribution of these metacarpals in the present work. 
Each finger is composed of three additional bones, the proximal, medial and distal phalanges. The joint between the metacarpal and the proximal phalange is the metacarpal-phalangeal joint (MCP). The next joint in the finger is the proximal interphalangeal joint (PIP) and the last joint is the distal interphalangeal joint (DIP). The interphalangeal joints are hinge joints which allow a small amount of hyperextension, while the MCP is more mobile.

The thumb is the most complex subsystem of the hand. The first metacarpal bone in the hand is the base bone of the thumb. This bone articulates with the trapezium in the first carpometacarpal (CMC) joint. The articular surfaces of this joint are both saddle shaped, and a loose but strong articular capsule joins the bones. This provides the joint with an axis of motion for flexion and extension as well as an axis for abduction and adduction. The looseness of the joint also allows for a small degree of passive rotary movement in the thumb, enabling opposition [Nette87].

Like the other fingers, the thumb also has an MCP joint connecting the metacarpal bone of the thumb to the proximal phalanx, and an interphalangeal joint (IP) that connects the proximal and distal phalanges. Notice that the MCP in the thumb is a hinge joint unlike the MCPs of the fingers, and that the thumb has no middle phalanx.

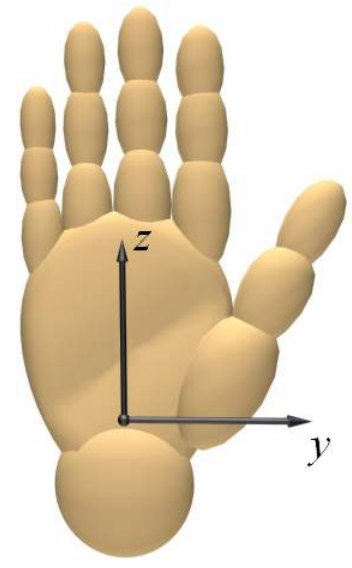

Figure 3: Palm Coordinates

\section{OUR MODEL}

Our representation of the hand consists of sixteen articulated rigid bodies, which represent the palm and the bones of the fingers and thumb. For convenience, and computational efficiency we model the palm as a single rigid body. This does not restrict our model unnecessarily, but we do have to introduce some addition rotational freedom in certain joints to compensate.

We place a reference right-handed coordinate frame $M_{\text {palm }}$ in the plane of the palm at its base, with the $z$-axis oriented up toward the fingers, the $y$-axis to the right toward the thumb, and the $x$-axis oriented out of the palm as in Figure 3.

\subsection{The Fingers}

The MCP joints of the fingers have two degrees of freedom about orthogonal axes. We begin with the fingers extended so that the $z$-axes of the fingers are parallel to the $z$-axis of the palm. Number the digits in the hand from one to five beginning with the thumb. Place a local coordinate frame $M_{i}$ at the base of the proximal phalanx of finger $i$ which is a parallel translation of $M_{\text {palm }}$. The rotations for spreading and bending the fingers occur about the $x$ and $y$ axes respectively.

The MCP joints of the fingers are not co-linear, see Figure 2, but the specific curve spanned by these joints depends on the hand in question. We position these joints along an arc representing the top of a typical palm. We will refine the specifications of this curve momentarily.

There is a small degree of twist about each finger's $z$-axis that occurs automatically in the fingers as they are spread [Caill82]. Since this twist amount is small, we found that by taking a median twist of each finger's coordinate frame to be the default position, we could obtain most of each finger's expressiveness.

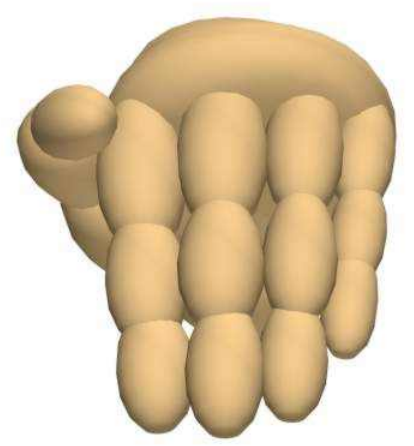

Figure 4: The convergence of the fingers when bent at the MCP.

Consider that if the $y$-axes of the bend rotations for all of the fingers are parallel, then when the fingers are bent $90^{\circ}$ at the MCP, the tips of the fingers will not converge, see Figure 4 . The significance of these twists is that they cause the fingers to converge as the MCP is bent. Also, if we cause the fingers to twist a bit more as they are spread, we can compensate somewhat for the absence of the metacarpals in the model. So, we correlate a $z$-axis twist of the finger with the spreading action with a range of $\left[-10^{\circ}, 10^{\circ}\right]$ about these default amounts.

The base twist amount depends on finger length. Let $L_{i}$ denote the resting lengths of each finger. Let $B_{i}$ denote the distance between finger $i$ and $i+1$ at the 
MCP, and let $P_{i}$ and $D_{i}$ denote the width of each finger at the PIP and DIP respectively. Since the twist angles of each finger relative to its neighbors are small, the following angles will yield approximate bend convergence in the fingers while avoiding collisions. See Figure 5.

$$
\begin{gathered}
\theta_{2}=\arcsin \left(\frac{2 B_{2}-\left(D_{2}+D_{3}\right)}{2 L_{2}}\right) \\
\theta_{4}=\arcsin \left(\frac{2 B_{4}-\left(D_{4}+D_{3}\right)}{2 L_{4}}\right) \\
\theta_{5}=\arcsin \left(\frac{2\left(B_{4}+B_{3}\right)-\left(D_{5}+2 P_{4}+P_{3}\right)}{2 L_{5}}\right)
\end{gathered}
$$

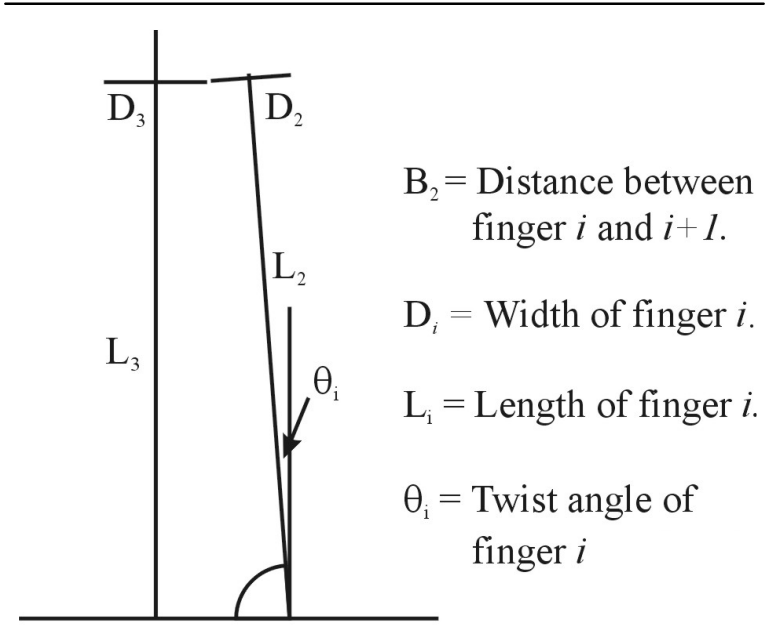

$\mathrm{B}_{2}$

Figure 5: Twist for finger convergence.

and $\theta_{3}=0$. We then position the MCP joints so that they lie on a curve to which these $y$-axes are tangential while preserving their base separations $B_{i}$.

Rotation about the MCP's $y$-axis yields the bending motion of the finger, while rotation about the $x$-axis spreads the finger. We apply the spread rotation first and then the bend rotation to the MCP. Unfortunately, the rotational limits for the spread axis are not uniform among the fingers. This is mostly due to the metacarpal bones in the palm. They are not lined up parallel to the $z$-axis of the palm, but rather they radiate out from the wrist at angles of approximately $-8^{\circ}, 0^{\circ}, 4^{\circ}, 14^{\circ}$ respectively for the index, middle, ring and pinky fingers, See Figure 6.

Once we rotate the coordinate frames of the MCPs about their $x$-axes by these amounts, the nonuniformities of the spread limits mostly disappear, and we can give each of these joints a rotational limit of $\left[-25^{\circ}, 30^{\circ}\right]$ about the $x$-axis. The bend action in the MCP is simpler with a flexion limit of $\left[0^{\circ}, 90^{\circ}\right]$ and about $15^{\circ}$ of hyperextension.

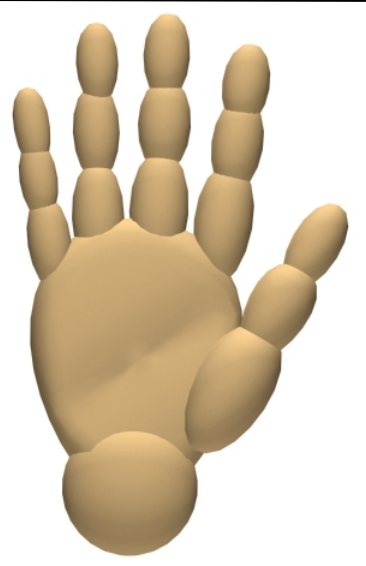

Figure 6: The default positions of the fingers.

The other two joints in the fingers are simpler hinge joints. They have one degree of freedom about their $y$-axes. Experimental evidence shows that in normal hands, the rotation limits of the PIP are generally $\left[0^{\circ}, 110^{\circ}-120^{\circ}\right]$. The DIP is similar but only has a range of motion of $\left[0,80^{\circ}-90^{\circ}\right]$. Both the PIP and the DIP also have the ability to hyperextend passively for about $15-25^{\circ}$ [Caill82].

\subsection{The Thumb}

This brings us to the thumb, the digit which gives our hand much of its expressiveness for applications such as ASL. The thumb is composed of three bones rather than four, but the connection between the trapezium and the metacarpal bone has far more freedom than any of the other joints in the hand.

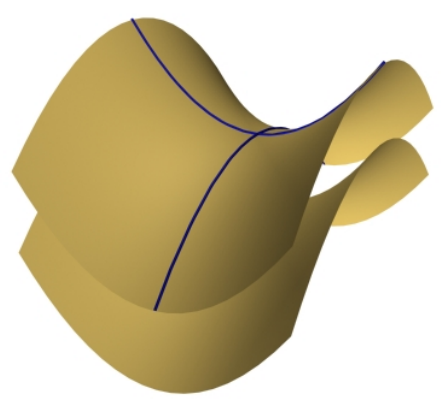

Figure 7: Two saddles and the parabolas

As mentioned previously, the thumb's carpometacarpal (CMC) joint is a saddle joint with highly complex rotations. Both the articular surfaces of the trapezium and the base of the metacarpal roughly form hyperbolic paraboloids. Such a joint can move along two orthogonal axes, but the foci of the resulting rota-tions are different, see Figure 7. Movement from a point on a hyperbolic paraboloid can proceed along two orthogonal parabolas, one oriented upwards, the other downwards as in the figure. Movement along the upward pointing parabola causes a rotation of the metacarpal with a focus inside the metacarpal itself, whereas move- 
ment along the other parabola causes a rotation of the metacarpal with a focus in the trapezium.

The articulation between the trapezium and the metacarpal is quite loose allowing this joint to rotate passively a bit. Since we didn't try to correlate rotations about different foci for this bone, we turned to the muscles of the thumb to determine a simple set of rotations to mimic the thumb's behavior. We place the center of these rotations inside the base of the metacarpal and give the joint some added rotational freedom to compensate for the lack of a true saddle joint here.

The largest muscle for the thumb is the adductor pollicis, which originates along the metacarpals of index and ring fingers, and inserts into the base of the proximal phalanx [Nette87]. The orientation of this muscle causes a lateral movement of the thumb across the palm upon contraction. Thus, one of the primary rotations of the thumb is about an axis approximately equal to the $z$-axis of the palm itself. The limits on the motion of the thumb about this axis are approximately $\left[-25^{\circ}, 90^{\circ}\right]$ including hyperextension. These rotations are measured from the plane of the palm.

With these rotational limits, however, we could not achieve a realistic convergence of the thumb and pinky fingers due to the absence of movement in the fourth and fifth metacarpal bones, and also due to the position of the focus of the rotation. To compensate, we gave the metacarpal bone a rotational limit of $120^{\circ}$ about this axis and allowed a small amount of additional rotational freedom in the pinky as described above.

The other motion of the thumb's metacarpal bone is the spread or extension of the thumb. This motion is caused by several muscles along the sides of the thumb, and serves to rotate the thumb away from the vertical axis of the palm. One interesting aspect of this rotation is that, depending on the position of the thumb, the action is caused by different sets of muscles, but this fact doesn't inconvenience us, as the functional result is the same in any case. This rotation has a functional range of approximately $\left[10^{\circ}, 90^{\circ}\right]$.

These two rotations reduce the motions of the thumb to rotations along the coordinate axes of a spherical coordinate system based inside the metacarpal. The adduction or bend of the thumb across the palm becomes the traditional rotation of $\theta$ about the vertical axis and the spread or extension of the thumb becomes the $\phi$ rotation away from this vertical axis.

The last aspect of the thumb's motion, which needs consideration, is a passive twist of the thumb correlated to the spread action. This twist places the thumb in certain positions of opposition. If one places the thumb in front of the palm and vertically in line with the index finger, and then spreads the thumb away from the palm, one will notice that the thumb passively rotates about its $z$-axis during the extension [Net97][Caill82].

To model this twist in the thumb, we placed a rotational correlation between the spread of the thumb and a twist about the thumb's local $z$-axis. This twist begins when the thumb is spread by approximately $60 \%$ of its range and encompasses approximately $45^{\circ}$ of rotation. We also scale this rotation in the case where the bend angle $\theta$ of the thumb is less than about $20^{\circ}$, in accordance with the above observation that the thumb doesn't twist as much during spread when the thumb is in these positions.

\subsection{Other Rotational Correlations}

Conveniently, this twist in the thumb is not the only correlation between rotations in the hand. We have already noted the correlation between the spread of the fingers and a small twist about their $z$-axes. In addition, we there is a far more noticeable correlation between the DIP and PIP joints in the fingers for hooking actions. As has been observed in previous works [Lands58][Rijpk91], the tendons that bend the DIP also bend the PIP. In most hooking actions of the fingers, such as during grasping, the DIP bend is approximately $2 / 3$ that of the PIP [Rijpk91]. We isolate this rotational correlation as an independent action called the hook of the finger.

There is also independent motion in the PIP itself, but unless the MCP of the finger is fully bent, this motion is difficult to achieve, so we restrict this motion to the case when the MCP is fully bent. Thus we can merge two independent aspects of the bending of the finger into one degree of freedom. The bend of the finger initiates with a rotation of the MCP about its $y$-axis until the MCP is bent $90^{\circ}$. The motion then proceeds by bending the PIP independently of the DIP from $0^{\circ}$ to $90-110^{\circ}$ depending on the flexibility of the hand in question.

In their recent paper on a general 3D model of the human hand[Ouhad99], Ouhaddi and Horain describe other rotational correlations in the hand which may be useful in future models. However, we found that the above correlations gave us the realism and simplicity needed for ASL.

\section{ROTATIONAL CONTROLS FOR THE HAND}

We take advantage of the rotational correlation in the user interface of our handshape transcription system. ASL linguists have identified several key independent motions in the fingers that are significant for ASL [Brent98][Lidde89][Sandl89], and fortunately, these motions correspond well to the rotational correlations specified above. The fingers have three independent degrees of freedom. The following list contains a description of each action. 
Each rotation is taken to vary along the full range of motion specified above.

1) Spread: Rotation of the MCP about its $x$ axis, with the small associated twist in the MCP about its $z$-axis.

2) Bend: For half of the bend range, this corresponds to rotation of the MCP about its $y$ axis and for the rest of the bend range, it corresponds to rotation of the PIP about its $y$-axis.

3) Hook: Bending the PIP and DIP simultaneously so that the PIP is $3 / 2$ of the DIP. Or in other words, the DIP $=2 / 3$ PIP

You will notice that the PIP is over specified by these actions, so for any [Spread, Bend, Hook] triple, we will set the PIP to the maximum rotation specified by the bend and the hook sliders.

The thumb has four independent degrees of freedom since the MCP and the DIP of the thumb are not correlated, and because the twist of the MCP is correlated to the other rotations of the CMC of the thumb.

1) Spread: The abduction or extension of the thumb away from the $z$-axis of the palm with the associated twist about the $z$-axis of the thumb.

2) Bend: The rotation of the thumb about the $z$-axis of the palm.

3) MCP Bend: Rotation of the thumb's MCP about it's $y$-axis.

4) DIP Bend: Rotation of the thumb's DIP about it's $y$-axis.

With these simple and intuitive controls, we can place the fingers of the palm in the positions necessary for American Sign Language, and animations of the handshapes look natural and convincing.

We implemented our version of the hand transcriber using a commercially available animation package, and using that package's scripting language to implement the rotational controls and constraints. The simplicity of its interface makes it feasible for non-experts to use it effectively. In the Appendix, we present a collection of pictures of a human hand making various ASL handshapes along with their representations in our model.

\section{CONCLUSIONS AND FUTURE WORK}

The above model provides an intuitive interface for shaping the positions of the fingers in the hand for ASL and many other applications. Initial user tests within the deaf community have been very promising, as it took only a few minutes for test subjects to familiarize themselves with the interface and then less than two minutes for them to transcribe a handshape such as ASL's " $m$ " or “ $t$ ".

This model still has one main deficiency that does not hinder its effectiveness for ASL. The absence of the metacarpal bones for the pinky and ring fingers makes it difficult to place the hand in certain configurations where the thumb is touching the pinky. So, we must make the palm deformable and add support for these two bones in the model and interface.

The next major improvement for this model would then be to transform the palm and fingers into deformable surfaces and to model the underlying bones and musculature of the hand to give it a far more realistic appearance as in [Wilhe97], [Gourr89] and [Schee97]. 


\section{REFERENCES}

[Badle99] Badler, N, Palmer, M, and Bindiganavale, R: Animation Control for Real-Time Virtual Humans, Communications of the ACM, Vol 42, August 1999, No. 8.

[Brent98] Brentari, D: A Prosodic Model of Sign Language Phonology. Cambridge, Massachusetts: MIT Press, 1998.

[Caill82] Cailliet, R, MD: Hand Pain and Impairment, Third Edition. F.A. Davis Company, 1982.

[Catmu72] Catmull, E: A System for Computer Generated Movies," Proc of the ACM National Conf, 1972, 422-431.

[Earns98] Earnshaw, R, Magnenat-Thalmann, N, Terzopoulos, D, Thalmann, D: Computer Animation for Virtual Humans, IEEE Comp Graphics App 18, 5, Sept-Oct 1998, 20-23.

[Girar90] Girard, M: Constrained Optimization of Articulated Animal Movement in Computer Animations, Making them Move (mechanics, control, and animation of articulated fingers), Morgan Kaufmann Publishers, 1990.

[Gourr89] Gourret, J, Magnenat-Thalmann, N, Thal-mann, D: Simulation of Object and Human Skin Deformations in a Grasping Task, ACM Siggraph Proc, 1989.

[Holt94] Holt, J., "Stanford Achievement Test - 8th Edition for Deaf and Hard of Hearing Students: Reading Comprehension Subgroup Results". http://www.gallaudet.edu/ cadsweb/satread.html

[Jaffe89] Jaffe, D: Evolution of Mechanical Fingerspelling Hands for People who are DeafBlind. On-Center-Technology Transfer News, 1: 1989

[Klima79] Edward Klima and Ursula Bellugi, The Signs of language. Harvard University Press, 1979.

[Lands58] Landsmeer, J: A Report on the Coordination of the Interphalangeal Joints of the Human Finger and its Disturbances. Acta Morphologica Neerlando-Scandinavica 2, 59-84, 1958.

[Lands63] Landsmeer, J: The Coordination of Finger-Joint Motions." Journal of Bone and Joint Surgery, vol 45-A, no 8, December 1963, pp 1654-1662.
[Lands55] Landsmeer, J: Anatomical and Functional Investigations on the Articulation of the Human Fingers. Acta Anatommica 24 supl 2, Vol 25, 1955, pp 1-69.

[Lidde89] Liddell, S, Johnson, R: American Sign Language: The Phonological Base. Sign Language Studies, 64, 1989, 195-277

[Nette87] Netter, F, MD: The CIBA Collection of Medical Illustrations, Volume 8, Musculoskeletal System. Ciba-Geigy Corporation, New Jersy, 1987.

[Ouhad99] Ouhaddi, H, Horain, P: Conception et adjustement d'un modèle 3D articulé de la main, Actes des $6^{\text {èmes }}$ journées du Groupe de Travail Réalité Virtuelle, Issy-les-Moulineaux, France, March 1998, pp. 83-90

[Rijpk91] Rijpkema, H, Girard, M: Computer Animation of Knowledge-Based Human Grasping, Computer Graphics, Vol 25, No 4, July 1991, pp 339-348.

[Sand189] Sandler, W: Phonological Representation of the Sign: Linearity and Nonlinearity in American Sign Language. Dordrecht, Holland, Foris Publications, 1989

[Schee97] Scheepers, F, Parent, R, Carlson, W, May, S: Anatomy-Based Modeling of the $\mathrm{Hu}-$ man Musculature. Proc. SIGGRAPH '97, 163172.

[Stern96] Sternberg, M, The American Sign Language Dictionary. Multicom, 1996. (CD $\mathrm{ROM})$

[Valli93] Clayton Valli and Ceil Luca, Linguistics of American Sign Language. Gallaudet University Press, 1993.

[Wilhe97] Wilhelms, J, Van Gelder, A: Anatomically Based Modeling, Proc. SIGGRAPH '97, 173-180

[Zhao94] Zhao, Z, Badler, N: Inverse Kinematics Positioning Using Nonlinear Programming for Highly Articulated Figures. ACM Transactions on Graphics, Vol. 13, No. 4, October 1994, 313-336. 
APPENDIX: Handshape Examples
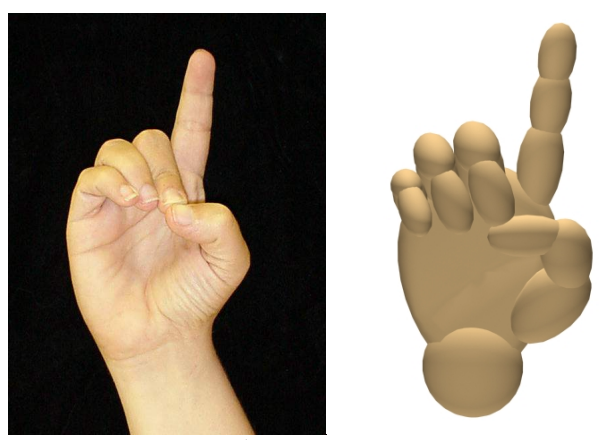

The Letter "D"

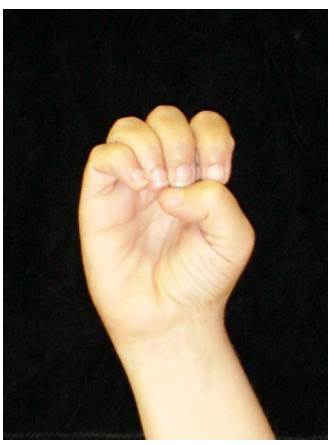

The Letter "E"
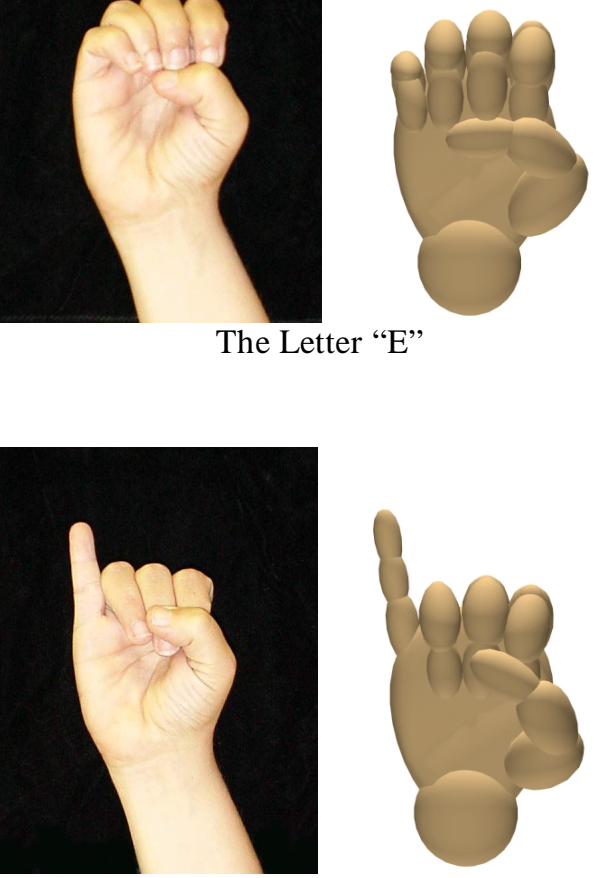

The Letter "I"
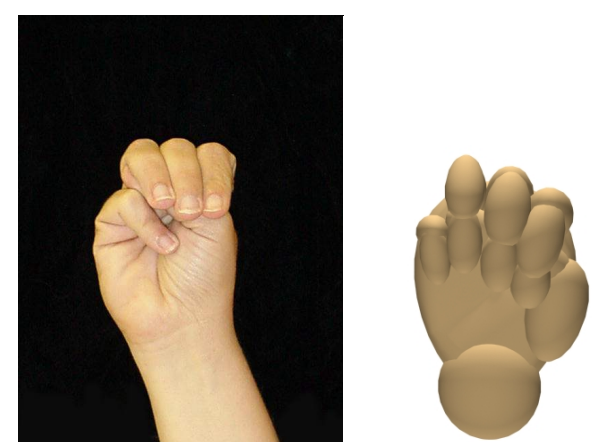

The Letter "M"
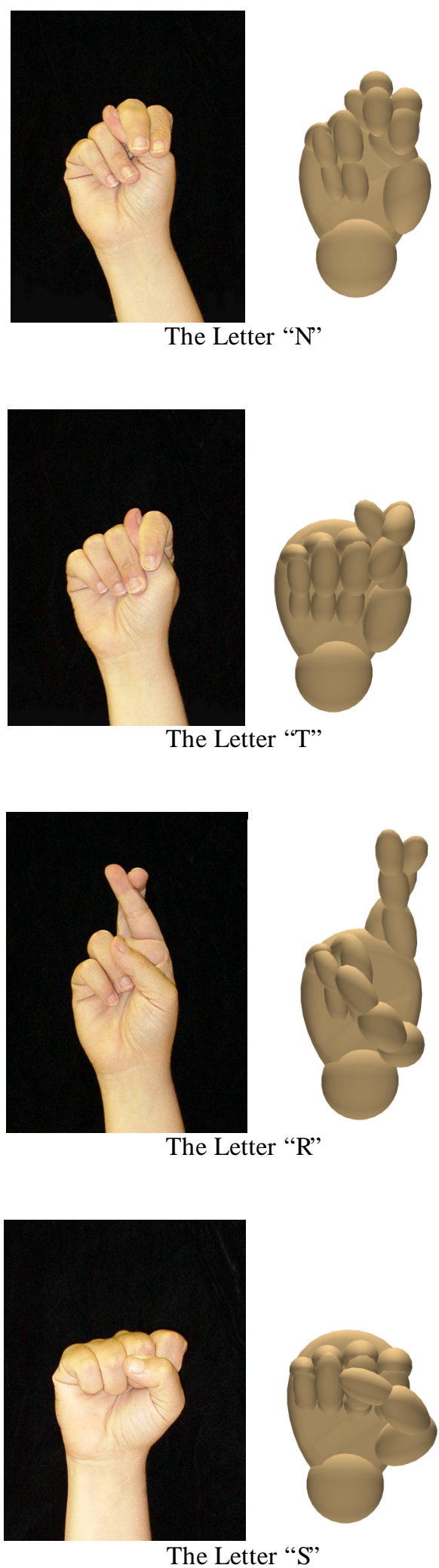